\title{
Circuit
}

Musiques contemporaines

\section{Chronique de disques}

\section{Michel Gonneville}

Volume 5, numéro 2, 1994

Espace Xenakis

URI : https://id.erudit.org/iderudit/902111ar

DOI : https://doi.org/10.7202/902111ar

Aller au sommaire du numéro

Éditeur(s)

Les Presses de l'Université de Montréal

ISSN

1183-1693 (imprimé)

1488-9692 (numérique)

Découvrir la revue

Citer ce compte rendu

Gonneville, M. (1994). Compte rendu de [Chronique de disques]. Circuit, 5(2), 88-96. https://doi.org/10.7202/902111ar

Ce document est protégé par la loi sur le droit d'auteur. L'utilisation des services d'Érudit (y compris la reproduction) est assujettie à sa politique d'utilisation que vous pouvez consulter en ligne.

https://apropos.erudit.org/fr/usagers/politique-dutilisation/
Cet article est diffusé et préservé par Érudit.

Érudit est un consortium interuniversitaire sans but lucratif composé de l'Université de Montréal, l'Université Laval et l'Université du Québec à Montréal. Il a pour mission la promotion et la valorisation de la recherche. https://www.erudit.org/fr/ 
«Forum 91 »: Cherney, Apparitions; Oña, Per Ivan Lermoliev; Gervasoni, Su un arco di bianco, Finsterer, Ruisselant. Le Nouvel Ensemble Moderne, dir. Lorraine Vaillancourt (UMMUS, série «Actuelles», UMM 106).

L'étiquette UMMUS /qui fait maintenant partie de l'écurie du distributeur et éditeur Analekta, ce qui devrait grandement favoriser la diffusion de ses produits) propose, deux ans après le fait, un enregistrement des œuvres des 
trois lauréats du Forum 91 du Nouvel Ensemble Moderne réalisé en direct lors du concert-gala qui clôturait cet événement, avec, en complément de programme, l'œuvre du compositeur québécois Brian Cherney, commandée pour cette occasion.

Bilan : un très beau disque! Qualité du répertoire, de l'interprétation, de la prise de son et de la réalisation technique: tout y est. Après audition, on comprendra que les enregistrements de Forum 91 ait connu plus de cinquante radiodiffusions dans une vingtaine de pays. Et pour ceux que les enregistrements en direct effraient, je dirai que l'énergie que dégage ce disque fait vite oublier les quelques bruits de pages tournées ou de toussotements du public.

Il fut un temps où les compositeurs justifiaient leur musique par le système de composition qu'ils employaient. Les notes de programme concernant les œuvres d'au moins deux des trois «jeunes» de ce disque font clairement référence à une attitude typique des récentes générations : la justification de leur musique (car il s'agit encore de cela) par le refus de tout système. Au-delà du discours du compositeur, nous avons avec ce disque la preuve que cette approche «intuitive " marche très bien et ne représente pas du tout une démission de la volonté de construction. Chez Oña et Gervasoni, il y a ce plaisir audible de jouer avec un petit nombre d'éléments clairement identifiables que l'on répète plusieurs fois en les variant de façon plus ou moins prononcée, que l'on superpose ou juxtapose plus ou moins abruptement avec le suivant ou le précédent, jouant avec la perception, l'attente de l'auditeur. Chez Oña, ces éléments sont parfois "culturellement chargés»/une gamme descendante, un accord parfait) et souvent plus brutalement dessinés; ils sont toujours poétiques chez Gervasoni, qui semble chercher sans cesse des couleurs impalpables, inouïes et raffinées. Finsterer, pour sa part, est moins radicalement "postmoderne " que ses deux collègues dans son attitude, dans son choix de matériau et dans sa façon de le composer, mais Ruisselant nous convainc malgré tout par la très grande énergie qui en émane de bout en bout, jusqu'à la cassure finale. Je disais que l'on avait affaire à de fortes personnalités : il faudra suivre les parcours du subtil Argentin, de l'Italien raffiné, de la grande et vigoureuse Australienne.

On respirait au Forum 91 du NEM l'excitation de la première édition d'un événement, voire une certaine exaltation, atmosphère que l'on pourra sans conteste retrouver, rendue palpable et magnifiquement maîtrisée grâce aux musiciens de l'Ensemble et à leur chef, dans ce très beau disque admirablement servi par la prise de son réalisée par l'équipe de la Société RadioCanada sous la direction inspirée de Laurent Major.

Si le Forum du NEM attire l'attention sur les jeunes compositeurs choisis par le jury international (et la cuvée de 1991 avait du caractère!), cela ne devrait 
pas faire oublier l'œuvre de Cherney, Apparitions, où la ligne de violoncelle solo, tout empreinte de la virtuosité impétueuse et des inflexions étranges typiques de l'excellent Claude Lamothe, parcourt un paysage de couleurs harmoniques et instrumentales habilement tissées. Métier du compositeur et talent des instrumentistes se rencontrent ici, tant le «beau son » développé par chacun des interprètes de l'Ensemble est mis en valeur. On pourra s'intéresser au rapport qu'entretiennent le style de Cherney et les poèmes du jeune Mallarmé auxquels les titres et sous-titres de l'œuvre se réfèrent: bien loin du radicalisme d'un Boulez sur ce plan, plus près d'un coloris debussyste, sans pour autant tomber dans le type de développement motivique qui afflige trop d'œuvres atonales d'outre-frontières... On sent la marque «résolument moderne» dans la matière constamment renouvelée de cette œuvre.

Ensemble d'Ondes de Montréal : Toussaint, Onde; Murail, Mach 2,5; Lesage, Les mystères de la clarté; Provost, Les Jardins suspendus; Messiaen, Oraison; Vivier, Pulau Dewata. Ensemble d'Ondes de Montréal avec Lise Daoust, flûte, et Serge Provost, piano (SNE, SNE-574-CD).

Ce disque témoigne éloquemment du "cas» de l'onde Martenot, c'est-àdire à la fois de ses grandeurs et de ses misères. Grandeurs de par le répertoire et les interprétations de grande qualité que les caractéristiques uniques de l'instrument ont inspirés, misères de par les limites mêmes que le développement technologique des ondes s'est trop longtemps imposées.

II me faut ici briser le cadre étroit de cette critique de disque pour esquisser un rapide portrait de l'étar de santé du Martenot. D'abord les rumeurs. Oui, l'Ensemble d'Ondes de Montréal s'est bien dissou après plusieurs années consacrées à la commande, à la création et à l'interprétation d'un répertoire unique présenté en concerts ici et en tournée à l'étranger; ce disque constitue en quelque sorte le chant du cygne de cette formation dont le visage aura varié avec les années et selon les projets. Eh oui, la classe d'ondes du Conservatoire de Québec a bien été fermée pour cause de compressions budgétaires, mais pas celle de Montréal, où une petite clientèle étudiante fréquente l'instrument, ne serait-ce parfois que comme discipline secondaire. Cette classe fait partie, avec celles de quelques conservatoires français, du petit nombre d'endroits au monde où cet instrument est encore enseigné. C'est là que Jean Laurendeau, infatigable professeur et animateur-initiateur, a formé bon nombre d'ondistes dont ceux qui constituaient l'EOM. Ce professeur et ses anciens élèves ont d'ailleurs fondé une Société pour le développement des ondes comportant un volet de "développement technique " qui n'a malheureusement pas eu beaucoup de temps et de moyens pour faire sa marque, mais assez cependant pour développer un prototype de l'instrument améliorant de façon notable le contrôle du timbre. 
Car non, les ondes Martenot ne sont pas mortes, et si le noyau de ses défendeurs reste petit, la poussière des musées n'est pas prête de recouvrir l'instrument du génial inventeur. On pourra s'étonner qu'au pays de l'IRCAM la chose ne soit pas venue plus tôt, mais les ateliers Martenot viennent enfin de sortir (et de lancer commercialement) une première version MIDI de leur produit, ce qui pourra ouvrir à celui-ci le monde de plus en plus raffiné de la génération et de la transformation sonore numérique et celvi de la microinformatique musicale.

Car si les gens intéressés reconnaissent sans hésiter l'apport unique de certaines caractéristiques des ondes comme la touche d'intensité, le ruban et le clavier mobile, plusieurs déplorent depuis longtemps sa palette timbrale d'un autre âge, si on la confronte avec celle que la récente évolution de la lutherie numérique a mise à la disposition des musiciens. De mon point de vue de compositeur (ou de celui d'une compositrice-ondiste comme Estelle Lemire), s'il doit y avoir recherche autour des ondes Martenot, il faudrait à la fois être respectueux et iconoclaste, conserver (pour pouvoir continuer à jouer le répertoire déjà écrit) et innover (pour justement rénover ce même répertoire et stimuler la création de nouvelles zones d'expressivité et donc de nouvelles œuvres). À mon avis, l'instrument ne survivra que par l'enthousiasme et l'opiniâtreté de ceux qui auront cette double attitude.

Cela dit, l'Ensemble nous propose dans ce disque SNE un répertoire franco-québécois dont on pourrait ranger les six œuvres entre deux pôles, le pôle mélodico-harmonique et le pôle timbral, selon les facettes de l'instrument qu'elles exploitent en priorité. L'arrangement de Pulau Dewata de Vivier et la belle Oraison de Messiaen se rattachent au premier pôle, et, à travers Lesage, Provost et Toussaint, on évolue vers l'approche presque purement électroacoustique de Tristan Murail.

Commençons par la déception de ce disque. Le Vivier, dont a pu entendre ailleurs des adaptations plus brillantes, plus incisives ou plus colorées, souffre ici de la trop longue réverbération des diffuseurs et de la polarisation de cette réverbération sur une fondamentale gênante, là d'un montage maladroit, ailleurs de certains timbres ou combinaisons de timbres mal choisis. Le Messiaen, de son côté, bénéficie d'une interprétation inspirée de sa mélodie, mais un timbre général un peu sourd et certaines obscurités qui nuisent par endroits à une bonne saisie de l'harmonie entachent le plaisir de l'auditeur.

Les autres pièces du disque m'incitent à être beaucoup plus positif. L'œuvre de lesage met en scène deux personnages dont l'un est constamment l'ombre de l'autre, la flûte étant plus souvent «réaliste», l'onde plus souvent impalpable, 
ces rôles étant habilement modulés à travers une série de courtes «situations » où l'approche mélodique dominante arrive à un bel équilibre avec la recherche plus purement sonore. Le propos est fascinant et fait "pardonner», ou, disons, intègre de façon cohérente, certains passages en octaves qu'ailleurs on trouverait de mauvais goût. Déconcertant postmodernisme...

L'œuvre de Provost - la plus longue du disque, une commande de l'EOM - met elle aussi en rapport les ondes Martenot avec un «outsider», le piano. Comme lesage, Provost cherche à combler la distance qui existe entre les deux sources sonores, l'acoustique et l'électroacoustique, plus précisément ici entre le piano surtout identifié au monde des hauteurs discrètes et tempérées et les ondes pouvant dépasser ce monde et explorer des univers sonores plus vastes. Le compositeur réussit à réduire leur étrangeté par toutes sortes de procédés efficaces /prolongements de notes, de sons harmoniques ou de cordes pincées du piano par les ondes, parfois avec de subtiles différences d'intonation ou grâce à un sens aigu de l'analyse du timbre, trame bruissante $d^{\prime}$ accompagnement des ondes pour le piano ou brouillages d'arpèges du piano par les ondes, etc.), tout en permettant ailleurs à certains "pianismes » ou "ondismes" de s'exprimer. Le tout s'organise selon une forme ample et riche, depuis les suspensions initiales jusqu'aux culminations ultérieures plus touffues.

Chez Toussaint, s'il reste encore quelques développements mélodiques, c'est la séduction sonore qui prend le dessus, le compositeur ayant habilement tiré parti de ce qui ailleurs devenait un défaut. La réverbération polarisée dont je parlais plus haut est ici formatrice, base harmonique et temporelle du matériau sur laquelle viennent se greffer, dans une respiration ample qui permet d'une façon très naturelle leur évolution, des points-bulles, une mélodie arabisante ou de grands balayages en glissando.

Enfin, avec Murail, on part pour un voyage fascinant à travers des strates sonores inouies, des masses vibrantes ou frémissantes de densité variable (comme les masses d'air que traverse le son d'un avion lointain); on entendra de grandes mouvances, des sifflements ou des cris entrecroisés d'animaux mystérieux, le tout charpenté par une grande conscience harmonique, de ce genre qui manque trop souvent aux œuvres électroacoustiques pures et dont plusieurs auditions ne finissent pas de révéler les subtilités. Encore là, certains défauts du Martenot lla réverbération déjà mentionnée et certains timbres style "ballon de plastique frotté »| ne devrait pas nous empêcher de considérer cette œuvre comme une contribution majeure au répertoire musical, dans le domaine de l'électroacoustique en direct.

P.S. : Il n'eût pas été inutile de mentionner dans le livret du disque les noms des ondistes solistes pour les œuvres de Messiaen (Jean Laurendeau) et de Lesage (Estelle Lemire)... 
«José Evangelista»: compositions d'Evangelista, Clos de vie, Piano concertant, Plume, un homme paisible, $\mathrm{O}$ Bali, Monodias espagñolas. Interprètes: Ensemble de la SMCQ sous la direction de direction Serge Garant; Orchestre de Radio-Canada et ensemble instrumental sous la direction de Lorraine Vaillancourt, Louise Bessette, piano, Pauline Vaillancourt, soprano, et Claude Lamothe, violoncelle (Salabert/Actuels, SCD 9102).

Les disques monographiques sont l'occasion idéale pour entrer en contacl intime avec l'univers d'un compositeur et se familiariser avec son langage et ses idées. C'est cette occasion que nous offrent les étiquettes UMMUS Isous la responsabilité de la Faculté de musique de I'Université de Montréall et Salabert Actuels (publiés par les Éditions Salabert, de Paris) en publiant l'une et l'autre un compact consacré à des compositeurs québécois.

Les œuvres de José Evangelista présentées ici témoignent toutes, sauf celle datant de 1974, de la manière d'écrire adoptée par le compositeur depuis 1982, une manière caractérisée, selon ses termes, par « une écriture monodique d'après laquelle toute la texture musicale se déduit d'une seule ligne mélodique sans le concours de l'harmonie ni du contrepoint " "I!. II est alors fascinant de découvrir, audition après audition, la richesse musicale à laquelle peut parvenir Evangelista à partir d'une telle restriction de son domaine de travail. La grande variété des ornementations et des arrangements en hétérophonie de ces «mélodies nucléaires » dont il se sert comme point de départ permet au compositeur d'en arriver à créer l'illusion d'une polyphonie ou encore d'en faire ressortir certaines harmonies latentes. Cette dimension harmonique potentielle est particulièrement réussie : les mélodies de base ont beau être construites sur les douze sons de la gamme chromatique, on perçoit régulièrement des polarisations modales sans cesse remises en mouvement, comme un jeu subtil de «modulations». À partir de ce donné, Evangelista peut ainsi, pour notre plus grand plaisir, se livrer à tout un travail de combinaisons des couleurs instrumentales et développer parfois certains idiomatismes, particulièrement audibles ici dans l'écriture pianistique.

Clos de vie, écrit à la mémoire de Claude Vivier, et Piano concertant constituent deux excellents exemples de l'art d'Evangelista. Tant de moments de ces œuvres peuvent charmer (comme le début du Canto - troisième mouvement de Piano concertant) ou intriguer (comme cette partie de Clos de vie où la mélodie de base devient très lente, entourée d'une nuée clairsemée de sons à la rythmique indécise). $O$ Bali, la pièce la plus récente du disque (1989), reste fidèle à cette manière tout en intégrant certains traits typiques de la musique balinaise : ornementations à plusieurs vitesses autour de la mélodie des flûtes, épisodes de tempi variés, sautes d'intensité, ponctuations brèves des instruments ornementateurs. L'hommage à Pulau Dewata (Bali, «l'île des dieux») s'inscrit en vérité dans un parcours éminemment personnalisé.
(1) Dans cette perspective, il es intéressant de relire l'article de José Evangelista, «Pourquoi composer de la musique monodique», paru dans le volume $1, n^{\circ} 2$ de Circuit (pp. 55-70). 
Les Monodias españolas doivent, pour leur part, être entendues un peu comme la célèbre orchestration que fit Webern du contrepoint final de L'Offrande musicale de Bach, y appliquant le concept de Klangfarbenmelodie que lui et ses confrères de la seconde école viennoise développèrent. Contrairement aux autres œuvres d'Evangelista, dont le titre ou certaines tournures modales pouvaient évoquer passagèrement certains univers musicaux « ethniques » sans que leur matériau de départ soit explicitement relié à ces univers, les mélodies de base ici sont clairement d'origine folklorique et la modalité de ces mélodies n'est pas du tout altérée. L'originalité d'Evangelista réside donc dans une technique d'articulation personnelle développée à travers des œuvres comme Piano concertant. On peut alors s'attarder à admirer ce qu'apporte cette magnifique écriture pianistique à la chanson d'origine, que l'on entend comme en filigrane. II faut souligner, ici comme dans le concerto, le superbe travail d'interprétation de Lovise Bessette qui allie la grande précision requise pour l'exécution d'une partition où l'ornementation est parfois touffue, à une sensibilité musicale perceptible dans la conduite des phrases et dans l'attention à la couleur du toucher.

De semblables éloges s'appliquent aux autres interprètes de ce disque et à leurs chefs, Lorraine Vaillancourt et le regretté Serge Garant. Secondée par Claude Lamothe, Pauline Vaillancourt manie habilement l'humour de Plume, un homme paisible, datant de 1974, mais dont certains passages annoncent les développements stylistiques ultérieurs de José Evangelista. II faut aussi ajouter un mot sur la qualité du livret de présentation (en quatre langues!). Celui-ci laisse la parole au compositeur qui nous présente dans un langage à la fois simple, direct et précis son itinéraire et ses œuvres.

"André Prévost : Sonates et improvisations » : compositions de Prévost, Sonate $n^{\circ} 1$ pour violoncelle et piano, Improvisation pour violon seul, Improvisation pour piano seul, Improvisation pour violoncelle seul, Improvisation pour voix et piano "Certains cris ", Improvisation pour alto, Sonate $n^{\circ} 2$ pour violoncelle et piano. Yuli Turovski, violoncelle, JeanEudes Vaillancourt, piano, Vladimir Landsman, violon, Louise-Andrée Baril, piano, Pauline Vaillancourt, soprano, Jutta Puchhammer-Sédillot, alto (étiquette UMMUS, série "Actuelles», UMM 103).

Le disque consacré à André Prévost permet, en sept œuvres pour solistes ou duos composées en 1962, 1976 et 1985, d'apprécier la continuité de vision, de ton et de langage manifestée par le compositeur durant ces vingttrois années en même temps que les différentes modalités d'existence de cette continuité. Parfois ostracisé par les modernes purs et durs de son propre pays - ce qui a pu contribuer à renforcer sa vision du monde -, Prévost n'en a pas 
moins continué à mener la barque de sa carrière, avec succès nombreux ef reconnaissances prestigieuses à la clé. Le livret de présentation, qui ici aussi laisse parler le compositeur, et la notice d'introduction de la musicologue Marie-Thérèse Lefebvre nous permettent de dégager certaines constantes de la démarche intérieure du compositeur, tout autant que les œuvres nous en font entendre la matérialisation artistique.

Une allusion à Beethoven, entre autres, dans la note sur la Sonate de 1985, n'est pas sans faire surgir l'image du combat maintes fois évoquée à propos du géant de la musique. Et Prévost, dans plusieurs des pièces ici présentées, propose une confrontation, un affrontement aux issues diverses entre deux forces, définies le plus généralement comme élément rythmique et élément mélodique. Et il le fait dans cet idiome sériel qui est le sien, proche de Berg, de Dutilleux et de tout un courant (européen autant qu'américain) qui n'a pas suivi les explorations des jeunes loups de la série intégrale et est demeuré attaché à une exploitation plus thématique de la série. Les rythmes cassés, les ostinati d'accompagnement s'opposent aux longues lignes rejoignant souvent dans leur parcours des paroxysmes sur le plan des hauteurs ou de l'intensité.

Les œuvres sont magistralement défendues par les interprètes de ce disque, où le violoncelliste Yuli Turovski est particulièrement mis en valeur (dans une des Improvisations et dans les deux Sonates, secondé par la présence très forte de Jean-Eudes Vaillancourt). La voix de Pauline Vaillancourt trouve juste ce qu'il faut de blancheur pour illustrer la gravité du poème de Certains cris et Louise-André Baril donne encore une fois une brillante démonstration de son talent, attentive, dirait-on, à contempler la densité de chaque accord, de chaque son qu'elle crée.

S'il m'a fallu quelques efforts pour vaincre certains préjugés ancrés depuis longtemps en moi contre certaines musiques de Prévost li'ai pu faire partie en mon temps des jeunes «modernes purs et durs» mentionnés plus haut, n'empêche que ses Fantasmes ont rythmé certains moments de mon adolescence et que je me plaisais encore il y a peu à écouter avec plaisir son Scherzo, tout bartokien qu'il fût...), ces efforts ont été récompensés principalement par I'Improvisation pour piano seul dont certains développements implacables par leur contrepoint (proche de musiques abstraites similaires chez Messiaen) et la menée formelle m'ont particulièrement séduit /ces clusters qui accompagnent l'énoncé initial du thème et qui se retrouvent seuls, encadrés de silence, pour clore l'œuvre: magnifique!). Je demeure réticent devant l'emploi lyrique des cordes à laquelle beaucoup d'autres auditeurs adhéreront cependant sans hésitation, et ce disque leur plaira évidemment beaucoup, compte tenu de la place accordée à ces instruments. 


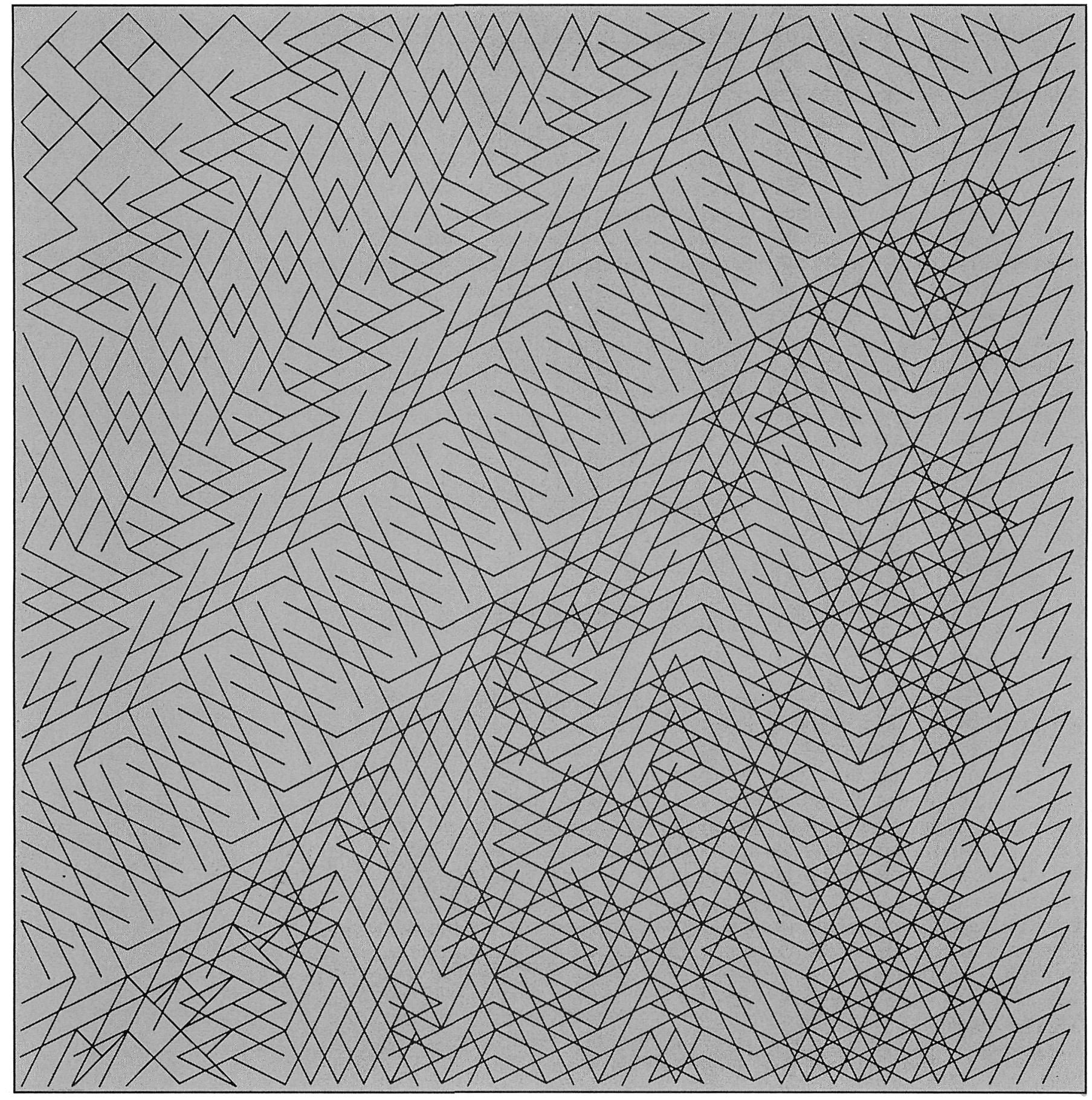

